\title{
The expressions of NEDD9 and E-cadherin correlate with metastasis and poor prognosis in triple-negative breast cancer patients
}

This article was published in the following Dove Press journal:

OncoTargets and Therapy

19 September 2016

Number of times this article has been viewed

\author{
Peng $\mathrm{Li}^{1}$ \\ Tingting Sun ${ }^{2}$ \\ Qingzhong Yuan' \\ Guozheng Pan' \\ Jian Zhang' \\ Diwen Sun' \\ 'Department of Breast and Thyroid \\ Surgery, ${ }^{2}$ Department of Clinical \\ Laboratory, Shengli Oilfield Central \\ Hospital, Dongying, Shandong, People's \\ Republic of China
}

Correspondence: Peng Li Department of Breast and Thyroid Surgery, Shengli Oilfield Central Hospital, No 3I, Jinan Rd, Dongying, Shandong, 257000, People's Republic of China

Tel +865468770901

Fax +865468717968

Email lipeng.good1987@163.com
Background: Neural precursor cell expressed, developmentally downregulated 9 (NEDD9), a member of Crk-associated substrate family, is involved in cancer cell adhesion, migration, invasion, and epithelial-mesenchymal transition. E-cadherin is a key event in the cellular invasion during the epithelial-mesenchymal transition mechanism. The aim of this study was to evaluate the association among NEDD9 expression, E-cadherin expression, and survival in triple-negative breast cancer (TNBC) patients.

Methods: NEDD9 and E-cadherin expressions were analyzed by immunohistochemistry in 106 TNBC patients and 120 non-TNBC patients. And the association of clinicopathological factors with survival was analyzed using Kaplan-Meier analysis and Cox regression in TNBC patients. Results: The results revealed that the rate of increased expression of NEDD9 and reduced expression of E-cadherin was significantly higher in TNBC group than that in non-TNBC group $(P<0.001$, both). Comparison of features between TNBC and non-TNBC groups showed that histological type $(P=0.026)$ and lymph node metastasis $(P=0.001)$ were significantly different. Correlation analysis showed that positive NEDD9 expression and negative E-cadherin expression were significantly correlated with lymph node metastasis and tumor-node-metastasis stage $(P<0.05)$. In addition, the enhanced NEDD9 expression was significantly associated with a reduced 5-year survival for TNBC patients (overall survival [OS]: $P=0.013$; diseasefree survival [DFS]: $P=0.021)$. Negative E-cadherin expression showed a significantly worse 5-year OS and DFS (OS: $P=0.011$; DFS: $P=0.012$ ). Multivariate analysis showed that lymph node metastasis (OS: $P=0.006$; DFS: $P=0.004)$, tumor-node-metastasis stage (OS: $P=0.012$; DFS: $P=0.001$ ), NEDD9 (OS: $P=0.046$; DFS: $P=0.022$ ), and E-cadherin (OS: $P=0.022$; DFS: $P=0.025$ ) independently predicted a poor prognosis of OS and DFS. Moreover, patients with NEDD9-positive/E-cadherin-negative expression had a significantly worse outcome than other groups (OS: $P=0.004$; DFS: $P=0.001$ ).

Conclusion: Our finding demonstrated the potential value of NEDD9 and E-cadherin expression levels as prognostic molecular markers and a target for new therapies for TNBC patients.

Keywords: NEDD9, E-cadherin, immunohistochemistry, EMT, prognosis, TNBC

\section{Introduction}

Triple-negative breast cancer (TNBC), which accounts for $10 \%-20 \%$ of all breast cancer patients, is a molecular subtype of breast cancer. ${ }^{1}$ It is defined by the lack of estrogen receptors, progesterone receptors, and lack of amplification of human epidermal growth factor receptor 2.,3 TNBC is generally more aggressive, with poorer prognosis due to high rates of metastatic disease than hormone receptor-positive genotypes. ${ }^{4,5}$ Therapy of TNBC has been a challenge to the doctors although having treatment 
with surgery, chemotherapy, and radiation. ${ }^{6-8}$ So far, many studies have focused on the identification of new biomarkers of TNBC, but the current biomarkers are less useful as prognostic markers. Therefore, new prognostic biomarkers that can predict therapeutic response and prognosis in TNBC patients are needed.

Neural precursor cell expressed, developmentally downregulated 9 (NEDD9), also called HEF1 and Cas-L, is best known for its roles in coordinating the FAK and SRC signaling cascades relevant to cell attachment, migration, and invasion. ${ }^{9-13}$ NEDD9, belonging to a member of Crk-associated substrate family, is a multidomain scaffolding protein. ${ }^{12}$ In recent years, studies have confirmed that NEDD9 contributes to cancer invasion and metastasis in several cancer types, such as breast cancer, ${ }^{14-16}$ melanoma, ${ }^{12}$ glioblastoma, ${ }^{17}$ lung cancer, ${ }^{18}$ and liver cancer. ${ }^{19}$ It is believed that NEDD9 regulates cell adhesion, migration, invasion, and epithelial-mesenchymal transition (EMT). ${ }^{15,20-22}$ Hence, NEDD9 expression changes have been identified as a biomarker in tumor aggressiveness.

The E-cadherin accumulated by the CDH1 gene located on16q22.1 chromosome is a cell adhesion molecule performing as cell invasion, proliferation, and a metastasis suppressor. ${ }^{23}$ E-cadherin is a key event in the cellular invasion during the EMT mechanism and in migration during the mesenchymalepithelial one. ${ }^{24}$ Retrospective studies suggested that E-cadherin is a novel prognostic factor for TNBC..$^{25,26}$

The aim of this study is to find out additional prognostic factors that can better identify TNBC with more aggressive behaviors. The current study examined the expressions of NEDD9 and E-cadherin in 106 TNBC patients, analyzed the possible correlation of NEDD9 and E-cadherin expressions with clinicopathological variables, and finally evaluated the association among NEDD9, E-cadherin expression, and survival in TNBC patients.

\section{Materials and methods}

\section{Clinical specimens and patient data}

From 2005 to 2010, a total of 226 breast cancer patients who were diagnosed and later underwent surgical resection at Shengli Oilfield Central Hospital were enrolled in this retrospective study. The tissue samples were collected from the tissue bank of the Department of Pathology in our hospital. A total of 226 cancer tissues were cut in wedge shapes and 57 normal tissues were cut at least $5 \mathrm{~cm}$ away from tumor margin. All of the patients in this study were diagnosed clinically and pathologically without having received preoperative chemotherapy or radiotherapy. Tumor stages and histopathology were defined based on the pathological tumor-node-metastasis (TNM) classification. Postsurgical chemotherapies, radiotherapy, and endocrinotherapy were confirmed according to the National Comprehensive Cancer Network guidelines. This study obtained consent from all patients and all procedures complied with the protocol and were approved by the Ethical Committee of Shengli Oilfield Central Hospital. All patients have follow-up records for 5 years by telephone or at the outpatient clinic till January 2010 or date of death.

\section{Immunohistochemistry and scoring}

Immunohistochemical study was performed as described before. ${ }^{27}$ Briefly, paraffin-embedded tissue sections were cut at $4 \mu \mathrm{m}$ thicknesses and deparaffinized for 2 hours at $60^{\circ} \mathrm{C}$ and then washed with distilled water after two and three changes of xylene and ethanol, respectively. Antigen retrieval was performed with microwave retrieval method, after which they were quenched endogenous peroxidase activity by incubation with $3 \%$ hydrogen peroxide. Sections were incubated with rabbit polyclonal antibody against human NEDD9 (1:100; Abcam, Cambridge, MA, USA) or monoclonal antibody against E-cadherin (clone NCH-38, dilution 1:200; Dako Denmark A/S, Glostrup, Denmark) overnight at $4{ }^{\circ} \mathrm{C}$, followed by incubation with horseradish peroxidase-conjugated secondary goat anti-rabbit antibody (Abcam) for 1 hour at room temperature. Sections were then washed with PBS and the antigen-antibody complex was visualized by using the Metal Enhanced DAB Substrate Kit (Thermo Fisher Scientific, Waltham, MA, USA).

Scoring of sections was separately assessed by two researchers blinded to the clinicopathological characteristics of the specimens. Expressions of NEDD9 and E-cadherin were evaluated according to the percentage of positivestained cells and staining intensity. The percentage of stained tumor cells on each section was counted and scored as follows: $0(<5 \%), 1(5 \%-25 \%), 2(26 \%-50 \%)$, and $3(>50 \%)$ accordingly. The intensity of NEDD9 and E-cadherin staining were scored as follows: 0 (no staining), 1 (weak staining), 2 (moderate staining), and 3 (strong staining). The scores of each case were multiplied to give a final score of $0,1,2$, $3,4,6$, or 9. The scores $0-3$ were regarded as low NEDD9 expression and 4-9 as high expression. E-cadherin expression was considered negative when scores were $0-1$ and as positive when the scores were 2-9.

\section{Statistical analysis}

Statistical analysis was performed using SPSS 17.0 software (SPSS Inc., Chicago, IL, USA). NEDD9 and E-cadherin expressions and clinical parameters were evaluated by the $\chi^{2}$ or Fisher's exact tests. Overall survival (OS) and disease-free 
survival (DFS) were plotted with the Kaplan-Meier method and the log-rank test was used to evaluate the differences. The association of clinicopathological factors with survival was analyzed using univariate Cox proportional hazards regression method. Prognostic factors for OS and DFS were determined by univariate and multivariate Cox analyses. A $P$-value $<0.05$ was considered significant.

\section{Results}

\section{The expression profiles of NEDD9 and E-cadherin in breast cancer patients}

The clinical characteristics of patients are summarized in Table 1. All patients were women and the mean age was 56 (range 28-79 years). The median follow-up was 27 months (range 9-60 months). The expressions of NEDD9 and E-cadherin in breast cancer tissues and nontumor breast tissues were evaluated by immunohistochemistry. High expression of NEDD9 was detected in 147/226 (65.0\%) of breast cancer tissues and 7/57 (12.3\%) nontumor breast tissues. Reduced E-cadherin expression was detected in
$114 / 226(50.4 \%)$ of breast cancer tissues and 8/57 (14.0\%) normal breast tissues. The rate of high NEDD9 expression and reduced E-cadherin expression was significantly higher in TNBC group than that in non-TNBC group $(P<0.001$, both). Comparison of features between TNBC and nonTNBC groups showed that histological type $(P=0.026)$ and lymph node $(\mathrm{LN})$ metastasis $(P=0.001)$ were significantly different (Table 1).

Immunohistochemical examination showed that NEDD9 was located predominantly in the cytoplasm in the nests of tumor cells and E-cadherin was intensely expressed on the membrane and weakly in the cytoplasm. The different intensities of staining are shown in Figure 1.

\section{The correlation of NEDD9 and E-cadherin expression with different clinicopathologic parameters}

We evaluated the correlation between NEDD9 expression level and a series of clinicopathological factors in TNBC group (Table 2). High NEDD9 expression was found to be

Table I Clinical characteristics of breast cancer patients

\begin{tabular}{|c|c|c|c|c|c|}
\hline Parameters & Total & TNBC $(n=106)(\%)$ & Non-TNBC $(n=120)(\%)$ & $\chi^{2}$ value & $P$-value \\
\hline Age (years) & & & & 0.698 & 0.404 \\
\hline$\leq 50$ & 94 & $4 \mathrm{I}(38.7)$ & $53(44.2)$ & & \\
\hline$>50$ & 132 & $65(61.3)$ & $67(55.8)$ & & \\
\hline Menopausal status & & & & 0.975 & 0.323 \\
\hline Premenopausal & 123 & $54(50.9)$ & $69(57.5)$ & & \\
\hline Postmenopausal & 103 & $52(49.1)$ & $51(42.5)$ & & \\
\hline Histological type & & & & 4.985 & 0.026 \\
\hline Ductal & 197 & $98(92.5)$ & $99(82.5)$ & & \\
\hline Lobular & 30 & $8(7.5)$ & $21(17.5)$ & & \\
\hline Size & & & & 2.852 & 0.091 \\
\hline$\leq 2 \mathrm{~cm}$ & 103 & $42(39.6)$ & $61(50.8)$ & & \\
\hline$>2 \mathrm{~cm}$ & 123 & $64(60.4)$ & $59(49.2)$ & & \\
\hline Pathological grade & & & & 1.178 & 0.278 \\
\hline I and II & 128 & $56(52.8)$ & $72(60.0)$ & & \\
\hline III & 98 & $50(47.2)$ & $48(40.0)$ & & \\
\hline LN metastasis & & & & 10.425 & 0.001 \\
\hline 0 & 130 & $49(46.2)$ & $81(67.5)$ & & \\
\hline$\geq 1$ & 96 & $57(53.8)$ & $39(32.5)$ & & \\
\hline TNM stage & & & & 2.173 & 0.140 \\
\hline 1 & 79 & $28(26.4)$ & $51(42.5)$ & & \\
\hline II & 83 & $43(40.6)$ & $40(33.3)$ & & \\
\hline III & 53 & $29(27.4)$ & $24(20.0)$ & & \\
\hline IV & 11 & $6(5.7)$ & $5(4.2)$ & & \\
\hline NEDD9 & & & & 17.707 & $<0.001$ \\
\hline Low expression & 79 & $22(20.8)$ & $57(47.5)$ & & \\
\hline High expression & 147 & 84 (79.2) & $63(52.5)$ & & \\
\hline E-cadherin & & & & 15.007 & $<0.001$ \\
\hline Negative expression & 114 & $68(64.2)$ & $46(38.3)$ & & \\
\hline Positive expression & 112 & $38(35.8)$ & $74(6 \mid .7)$ & & \\
\hline
\end{tabular}

Abbreviations: LN, lymph node; NEDD9, neural precursor cell expressed, developmentally downregulated 9; TNBC, triple-negative breast cancer; TNM, tumor-nodemetastasis. 

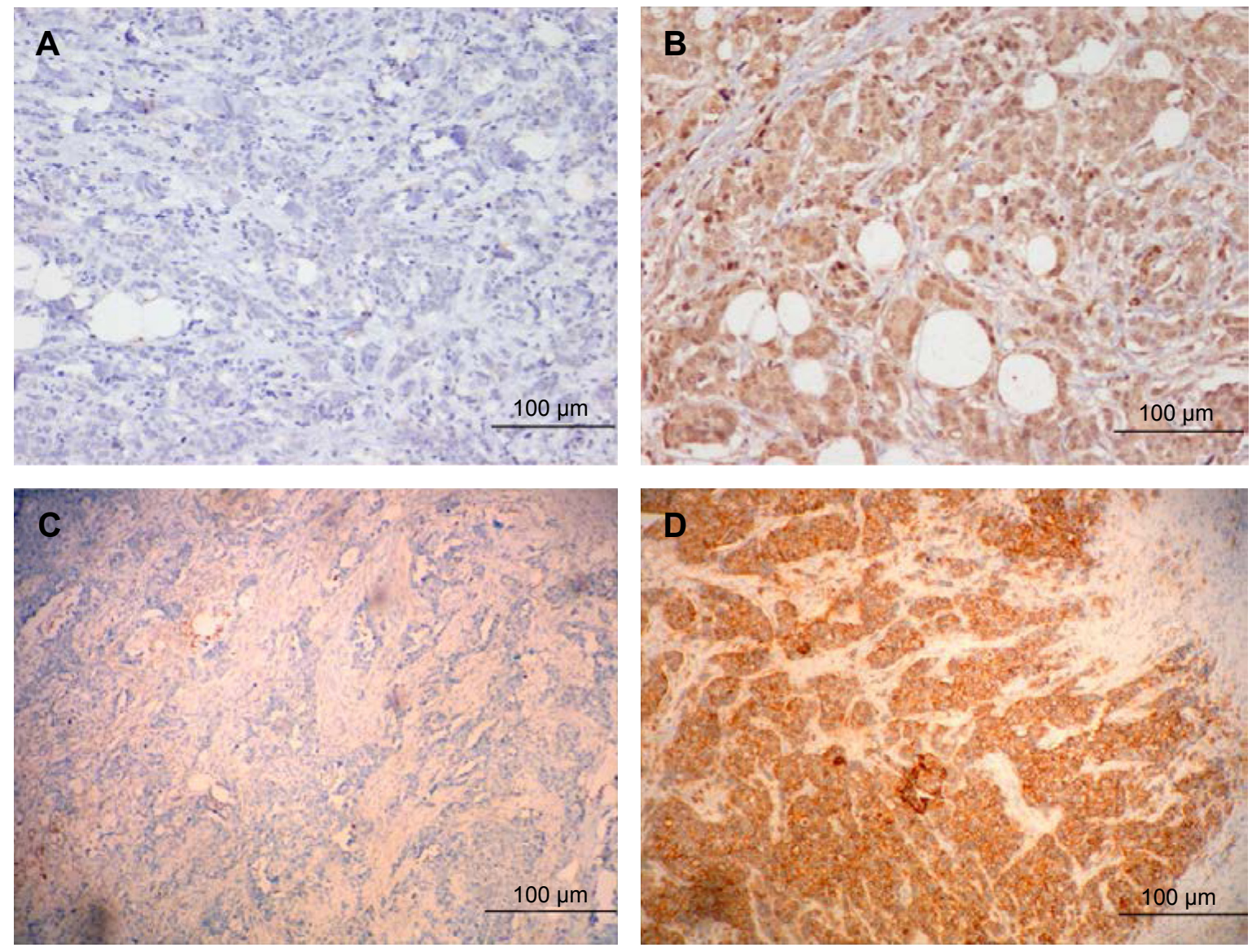

Figure I Representative immunohistochemical staining of NEDD9 and E-cadherin in TNBC: (A) Low expression of NEDD9; (B) High Expression of NEDD9 in the cytoplasm of breast carcinoma cells; (C) Negative expression of E-cadherin; and (D) Positive expression of E-cadherin on the membrane of breast carcinoma cells. A, B, C, and $\mathbf{D}$ is $\times 200$ magnification.

Abbreviations: NEDD9, neural precursor cell expressed, developmentally downregulated 9; TNBC, triple-negative breast cancer.

significantly correlated with LN metastasis and TNM stage $(P<0.05)$, but not correlated with other clinicopathological parameters, such as menopausal status, age, size, pathological grade, and histological type.

Expression of E-cadherin-negative correlated with LN metastases and TNM stage $(P<0.05)$. E-cadherin expression did not correlate with age, size, pathological grade, menopausal status, or histological type (Table 3).

\section{NEDD9 and E-cadherin expressions correlate with OS and DFS}

The 5-year OS and DFS of TNBC patients based on NEDD9 and E-cadherin expressions are shown in Figure 2. Notably, high expression of NEDD9 was significantly associated with a reduced 5-year survival for TNBC patients (OS: $P=0.013$; DFS: $P=0.021$ ). The 5 -year OS was $63.1 \%$ in TNBC patients displaying high NEDD9 expression, while it was $90.9 \%$ in the low NEDD9 expression group. Similarly, the 5-year DFS of high NEDD9 expression was $58.3 \%$ in TNBC patients, while it was $81.8 \%$ in the low expression of NEDD9 group.
Meanwhile, TNBC with negative E-cadherin expression showed a significantly worse 5-year OS and DFS (OS: $P=0.011$; DFS: $P=0.012$ ). These data suggest that high NEDD9 and negative E-cadherin expressions are poor prognostic indicators for TNBC patients. Furthermore, we evaluated the predicative value of NEDD9 by the Cox regression analysis. In univariate analysis, menopausal status (OS: $P=0.012$; DFS: $P=0.004$ ), grade (OS: $P=0.001$; DFS: $P=0.002)$, LN metastasis (OS: $P<0.001$; DFS: $P<0.001$ ), TNM stage (OS: $P<0.001$; DFS: $P<0.001$ ), NEDD9 (OS: $P=0.026$; DFS: $P=0.042$ ), and E-cadherin (OS: $P=0.016$; DFS: $P=0.015$ ) were significantly associated with worse survival. And the multivariate analysis showed that $\mathrm{LN}$ metastasis (OS: $P=0.006$; DFS: $P=0.004)$, TNM stage (OS: $P=0.012$; DFS: $P=0.001$ ), NEDD9 (OS: $P=0.046$; DFS: $P=0.022$ ), and E-cadherin (OS: $P=0.022$; DFS: $P=0.025$ ) independently predict poor prognosis of OS and DFS (Table 4).

We detected the outcomes of NEDD9-positive/ E-cadherin-negative, NEDD9-negative/E-cadherin-positive, NEDD9-positive/E-cadherin-positive, and NEDD9-negative/ 
Table 2 Correlation between NEDD9 expression and clinicopathological features of TNBC

\begin{tabular}{|c|c|c|c|c|}
\hline \multirow[t]{2}{*}{ Parameters } & \multicolumn{2}{|c|}{$\begin{array}{l}\text { NEDD9 } \\
\text { expression }\end{array}$} & \multirow[t]{2}{*}{$\begin{array}{l}t / \chi^{2} \\
\text { value }\end{array}$} & \multirow[t]{2}{*}{$P$-value } \\
\hline & Low & High & & \\
\hline Age (years) & & & 0.537 & 0.464 \\
\hline$\leq 50$ & 10 & 31 & & \\
\hline$>50$ & 12 & 53 & & \\
\hline Menopausal status & & & 1.515 & 0.218 \\
\hline Premenopausal & 15 & 45 & & \\
\hline Postmenopausal & 7 & 39 & & \\
\hline Histological type & & & 0.095 & 0.758 \\
\hline Ductal & 20 & 78 & & \\
\hline Lobular & 2 & 6 & & \\
\hline Size & & & 2.584 & 0.108 \\
\hline$\leq 2 \mathrm{~cm}$ & 12 & 30 & & \\
\hline$>2 \mathrm{~cm}$ & 10 & 54 & & \\
\hline Pathological grade & & & 2.625 & 0.105 \\
\hline I and II & 15 & 41 & & \\
\hline III & 7 & 43 & & \\
\hline LN metastasis & & & 5.384 & 0.020 \\
\hline 0 & 15 & 34 & & \\
\hline$\geq 1$ & 7 & 43 & & \\
\hline TNM stage & & & 10.016 & 0.014 \\
\hline I & 12 & 16 & & \\
\hline II & 6 & 37 & & \\
\hline III & 4 & 25 & & \\
\hline IV & 0 & 6 & & \\
\hline
\end{tabular}

Abbreviations: LN, lymph node; NEDD9, neural precursor cell expressed, developmentally downregulated 9; TNBC, triple-negative breast cancer; TNM, tumor-node-metastasis.

Table 3 Correlation between E-cadherin expression and clinicopathological features of TNBC

\begin{tabular}{|c|c|c|c|c|}
\hline \multirow[t]{2}{*}{ Parameters } & \multicolumn{2}{|c|}{ E-cadherin expression } & \multirow{2}{*}{$\begin{array}{l}t / \chi^{2} \\
\text { value }\end{array}$} & \multirow[t]{2}{*}{$P$-value } \\
\hline & Negative & Positive & & \\
\hline Age (years) & & & 0.084 & 0.772 \\
\hline$\leq 50$ & 27 & 14 & & \\
\hline$>50$ & 41 & 24 & & \\
\hline Menopausal status & & & 0.054 & 0.817 \\
\hline Premenopausal & 42 & 18 & & \\
\hline Postmenopausal & 26 & 10 & & \\
\hline Histological type & & & 0.443 & 0.506 \\
\hline Ductal & 62 & 36 & & \\
\hline Lobular & 6 & 2 & & \\
\hline Size & & & 0.001 & $0.98 I$ \\
\hline$\leq 2 \mathrm{~cm}$ & 27 & 15 & & \\
\hline$>2 \mathrm{~cm}$ & 41 & 23 & & \\
\hline Pathological grade & & & $0.14 \mid$ & 0.708 \\
\hline I and II & 35 & 21 & & \\
\hline III & 33 & 17 & & \\
\hline $\mathrm{LN}$ metastasis & & & 4.873 & 0.027 \\
\hline 0 & 26 & 23 & & \\
\hline$\geq 1$ & 42 & 15 & & \\
\hline TNM stage & & & 9.247 & 0.022 \\
\hline 1 & 13 & 15 & & \\
\hline ॥ & 26 & 17 & & \\
\hline III & 24 & 5 & & \\
\hline IV & 5 & I & & \\
\hline
\end{tabular}

Abbreviations: LN, lymph node; TNBC, triple-negative breast cancer; TNM, tumor-node-metastasis.
E-cadherin-positive (Figure 3). And we found that there were significant differences in OS and DFS comparisons (OS: $P=0.004$; DFS: $P=0.001$ ). Patients with NEDD9-positive/ E-cadherin-negative had a significantly worse outcome than other groups.

\section{Discussion}

TNBC is an invasive molecular subtype without specific target therapy. ${ }^{28,29}$ Compared to the other subtypes of tumor, TNBC is biologically more aggressive and is associated with higher recurrence rates and higher frequency of metastasis and poor OS in 5 years. ${ }^{30-32}$ Moreover, TNBC is a heterogeneous disease. Thus, new markers to identify prognosis are necessary to allow a better characterization and appropriate therapeutic strategies for TNBC.

Tumor invasiveness often requires EMT during which cells lose lateral attachments and become more motile. In recent several decades, EMT in the role of epithelial tumor progression has been widely studied..$^{33}$ One symbol of EMT is downregulation of E-cadherin acting as a cell-cell adhesion protein, resulting in instability of the adherens junctions that connect cells. ${ }^{34}$ By undergoing this transition, epithelial cells gradually lose their epithelial markers, such as E-cadherin, laminin-1, and cytokeratin, and express the mesenchymal markers, such as $\mathrm{N}$-cadherin, $\beta$-catenin, and vimentin. As a consequence, epithelial tumor cells acquire the mesenchymal characteristic with increased invasiveness and migration, and ultimately lead to metastasis. ${ }^{35-37}$ Accordingly, epithelial tumor cells get enhanced invasion and migration potential, and ultimately would metastasize to distant sites. ${ }^{37}$ Mani et al found that activation of EMT in breast cancer epithelial cells results in expression of mesenchymal traits and markers. ${ }^{38}$ Mani found that FOXC2 high expression was induced in cells undergoing EMT progress, and FOXC2 was associated with the aggressive basal-like human breast cancers. ${ }^{39}$ Moreover, as an EMT inducer, LBX1 was shown to be upregulated in the triple-negative basal-like breast cancer. ${ }^{40}$ These studies demonstrated that EMT played a key role in the invasion and metastasis of TNBC.

NEDD9 is a focal adhesion scaffolding protein related to p130 Crk-associated substrate. Several studies have proved that NEDD9 is connected with migration, invasion, and metastasis of cancer cells. Fashena et al found that NEDD9 induced cell motility and spreading in MCF7 cell lines, ${ }^{11}$ and Izumchenko et al reported that the NEDD9-null genetic background significantly reduced the mammary tumor cells initiation in the MMTV-polyoma virus middle $\mathrm{T}$ genetic model. ${ }^{14}$ Kong et al determined that NEDD9 was highly 
A

NEDD9

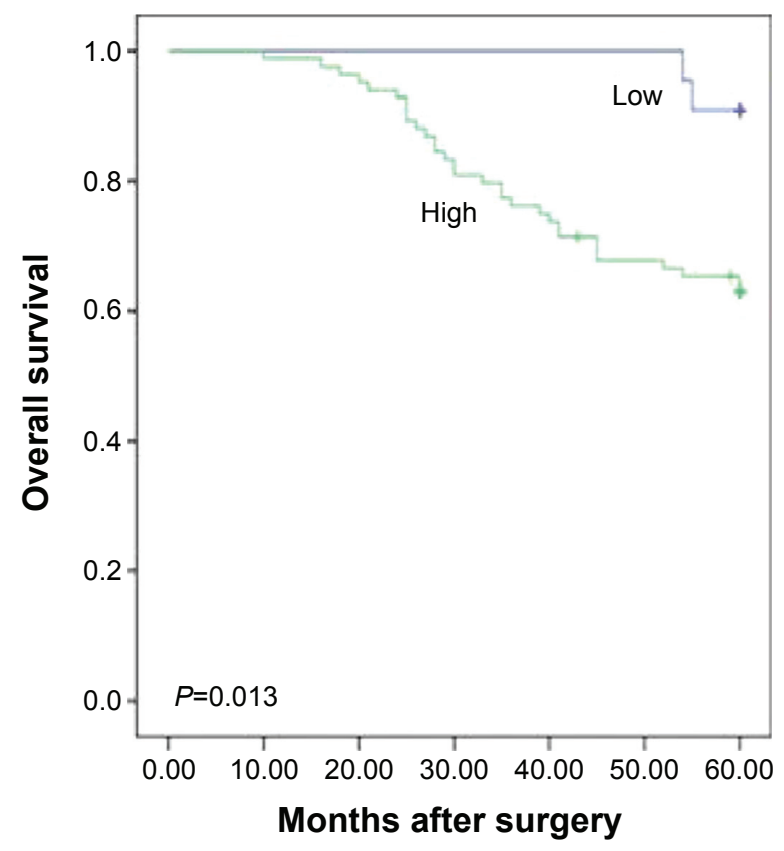

C

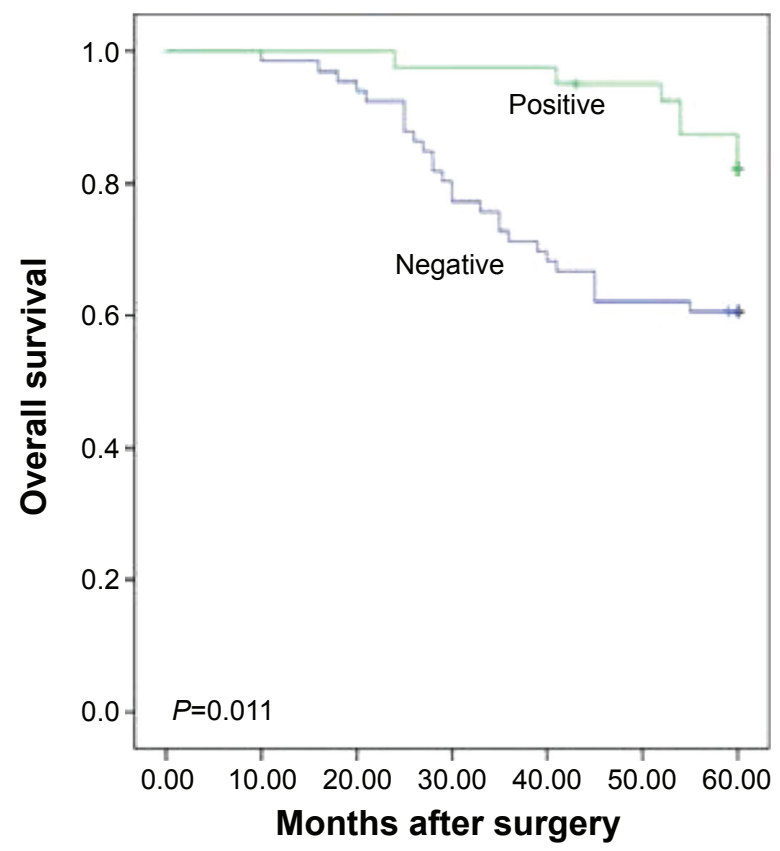

B

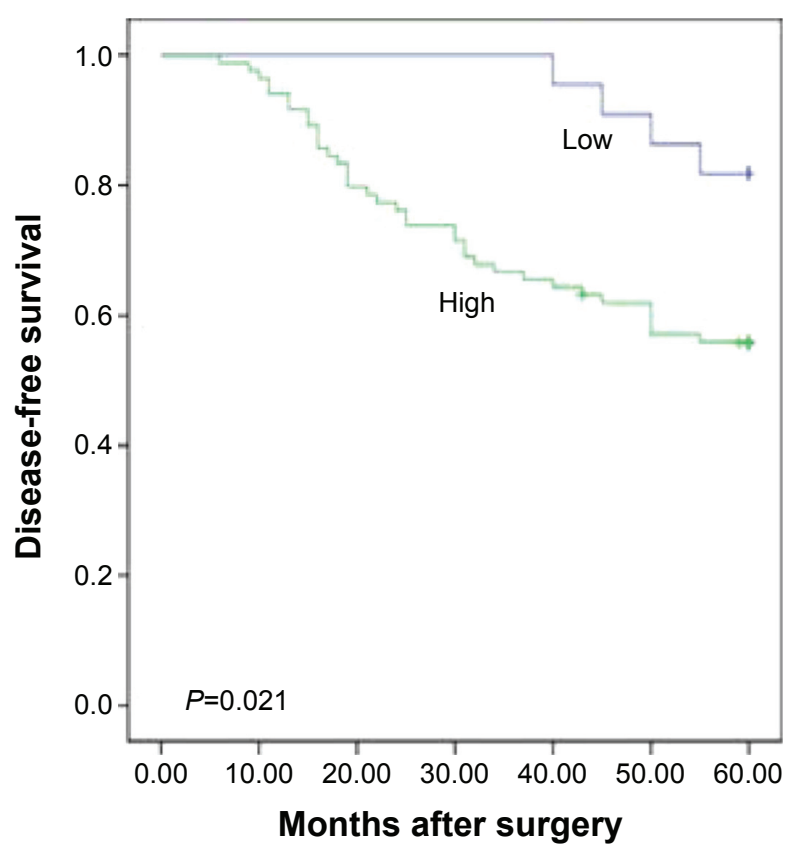

D

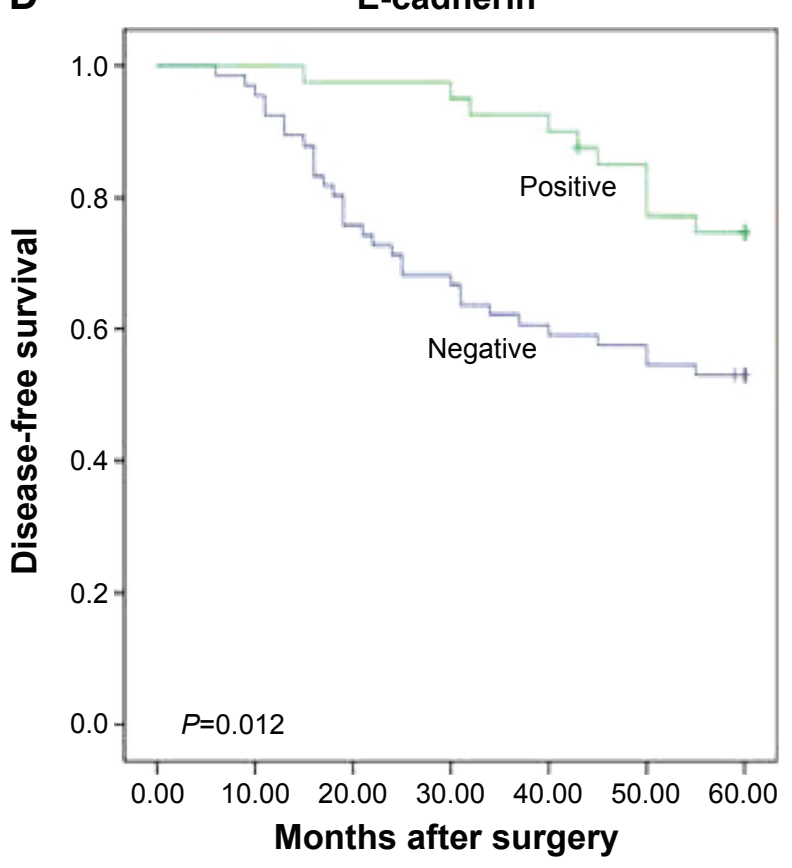

Figure 2 Kaplan-Meier survival illustrating the significance of NEDDD9 expression in comparison with E-cadherin expression in TNBC.

Notes: (A) OS curve of the TNBC patients based on NEDD9 expression. (B) DFS curve of the TNBC patients based on NEDD9 expression. (C) OS curve of the TNBC patients based on E-cadherin expression. (D) DFS curve of the TNBC patients based on E-cadherin expression. All $P<0.05$.

Abbreviations: NEDD9, neural precursor cell expressed, developmentally downregulated 9; TNBC, triple-negative breast cancer.

expressed in human breast cancers and promoted migration and invasion in TNBC. Moreover, NEDD9 overexpression induced EMT process and promoted their interactions in vivo with the E-cadherin promoter. ${ }^{15}$ Hence, NEDD9 seems to be related with tumor progression and metastasis and also a regulator in the EMT. In this study, our results revealed that
NEDD9 was highly expressed in most breast cancer tissues compared with normal tissues. And, we showed that the rate of high NEDD9 expression was significantly higher in TNBC than that in non-TNBC group. Moreover, the expression of NEDD9 in TNBC patients was significantly associated with the LN metastasis and TNM stage. Our results were similar to 
Table 4 Univariate and multivariate analysis of clinicopathlogical parameters with OS and DFS by Cox proportional hazards regression

\begin{tabular}{|c|c|c|c|c|c|c|}
\hline \multirow[t]{2}{*}{ Factors } & \multicolumn{3}{|c|}{ Univariate } & \multicolumn{3}{|c|}{ Multivariate } \\
\hline & OR & $95 \% \mathrm{Cl}$ & $P$-value & OR & $95 \% \mathrm{Cl}$ & $P$-value \\
\hline \multicolumn{7}{|l|}{ OS } \\
\hline Age & 0.980 & $0.956-1.005$ & 0.115 & & & \\
\hline Size & 1.605 & $0.764-3.374$ & 0.212 & & & \\
\hline Histological type & 0.730 & $0.175-3.049$ & 0.666 & & & \\
\hline Menopausal status & 0.384 & $0.183-0.808$ & 0.012 & 0.348 & $0.112-1.085$ & 0.069 \\
\hline Pathological grade & 3.694 & $1.716-7.956$ & 0.001 & 1.549 & $0.667-3.598$ & 0.309 \\
\hline $\mathrm{LN}$ metastasis & 11.972 & $3.647-39.300$ & $<0.001$ & 7.193 & $1.747-29.619$ & 0.006 \\
\hline TNM stage & 4.087 & $2.554-6.566$ & $<0.001$ & 2.222 & $1.191-4.146$ & 0.012 \\
\hline NEDD9 & 5.055 & $1.209-21.139$ & 0.026 & 4.554 & $1.028-20.170$ & 0.046 \\
\hline E-cadherin & 0.357 & $0.155-0.824$ & 0.016 & 0.355 & $0.146-0.883$ & 0.022 \\
\hline \multicolumn{7}{|l|}{ DFS } \\
\hline Age & 0.976 & $0.954-0.999$ & 0.139 & & & \\
\hline Size & 1.244 & $0.647-2.394$ & 0.512 & & & \\
\hline Histological type & 0.280 & $0.038-2.036$ & 0.208 & & & \\
\hline Menopausal status & 0.372 & $0.188-0.734$ & 0.004 & 0.453 & $0.168-1.225$ & 0.119 \\
\hline Pathological grade & 2.910 & $1.493-5.669$ & 0.002 & 1.153 & $0.574-2.318$ & 0.689 \\
\hline LN metastasis & 11.419 & $4.047-32.222$ & $<0.001$ & 4.760 & $1.636-13.844$ & 0.004 \\
\hline TNM stage & 4.287 & $2.685-6.845$ & $<0.001$ & 2.630 & $|.48|-4.670$ & 0.001 \\
\hline NEDD9 & 2.928 & I.040-8.245 & 0.042 & 3.604 & $1.205-10.779$ & 0.022 \\
\hline E-cadherin & 0.413 & $0.202-0.843$ & 0.015 & 0.415 & $0.192-0.897$ & 0.025 \\
\hline
\end{tabular}

Abbreviations: $\mathrm{Cl}$, confidence interval; DFS, disease-free survival; LN, lymph node; NEDD9, neural precursor cell expressed, developmentally downregulated 9; OS, overall survival; TNM, tumor-node-metastasis; OR, odds ratio.

A

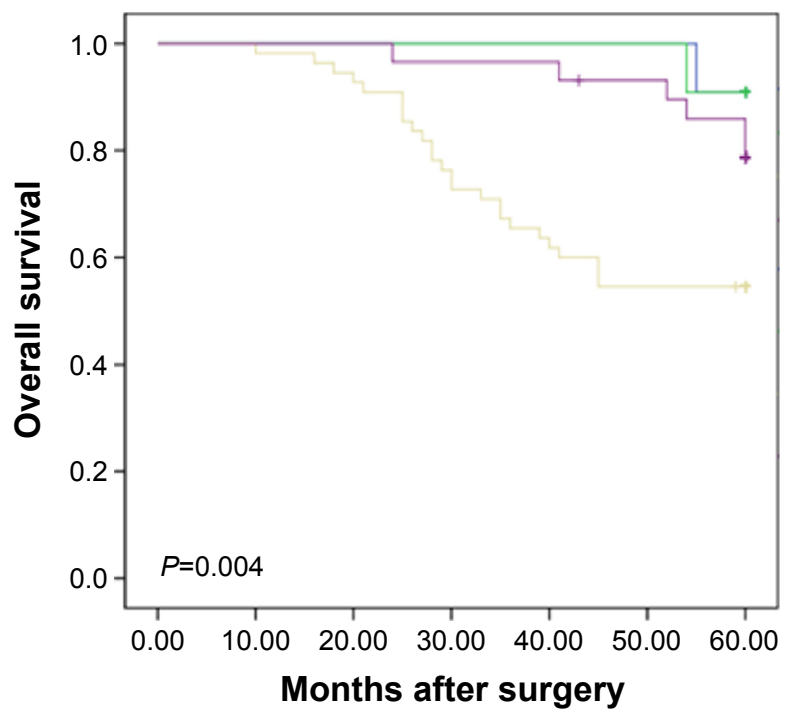

B

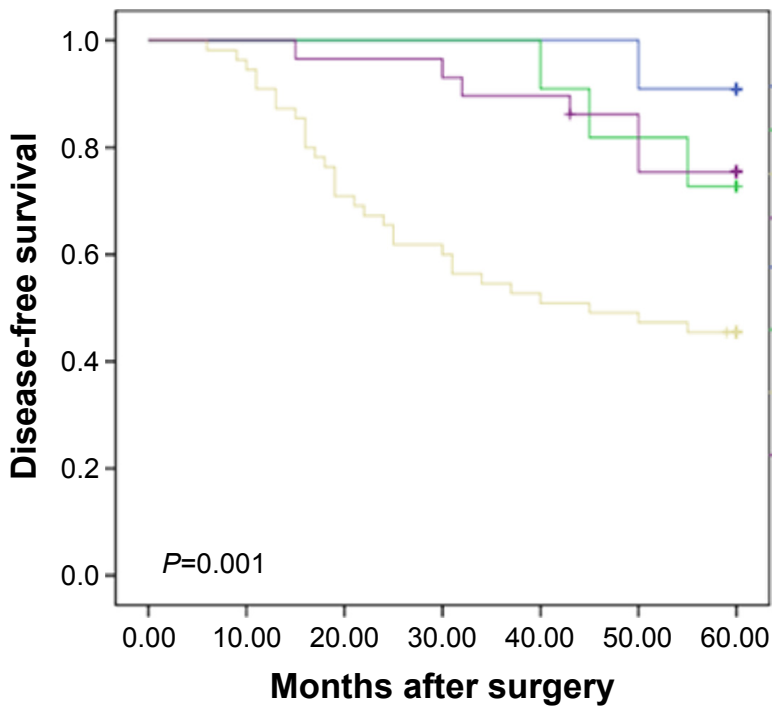

\begin{tabular}{|llll} 
& & Expression \\
& & & \\
NEDD9-negative/ & NEDD9-positive/ & + NEDD9-negative/E-cadherin- & NEDD9-positive/E-cadherin- \\
E-cadherin-negative & E-cadherin-negative & negative-censored & negative-censored \\
$\Omega$ NEDD9-negative/ & NEDD9-positive/ & + NEDD9-negative/E-cadherin- & + NEDD9-positive/E-cadherin- \\
E-cadherin-positive & E-cadherin-positive & positive-censored & positive-censored \\
\hline
\end{tabular}

Figure 3 Kaplan-Meier curve with univariate analyses (log-rank) for patients with NEDD9-positive/E-cadherin-negative, NEDD9-negative/E-cadherin-positive, NEDD9positive/E-cadherin-positive, and NEDD9-nagative/E-cadherin-positive.

Notes: (A) OS curve of TNBC patients based on coexpression of NEDD9 and E-cadherin. (B) DFS curve of TNBC patients based on coexpression of NEDD9 and E-cadherin. All $P<0.05$.

Abbreviation: NEDD9, neural precursor cell expressed, developmentally downregulated 9. 
these studies and suggested that high expression of NEDD9 may be involved in progression and EMT regulation of TNBC. The prognostic value of NEDD9 had been validated in other tumors: high expression of NEDD9 was an independent predictive factor in lung cancer ${ }^{41}$ and hepatocellular carcinoma. ${ }^{42}$ Consistently, we found that the TNBC patients with a high NEDD9 expression were associated with a shorter OS and DFS; furthermore, both univariate and multivariate Cox regression revealed that high NEDD9 expression was an independent poor prognostic factor. Consequently, it is worthy to further evaluate its value as a prognosis marker for the benefit of TNBC patients.

E-cadherin is one member of the cadherin superfamily. As a tumoral suppressor protein, E-cadherin has the ability to block the uncontrolled proliferation and cellular differentiation toward a malignant phenotype. ${ }^{23}$ As a result, complete or partial loss of E-cadherin expression is involved in tumor invasion and metastasis process. ${ }^{23}$ The process of breast cancer started with the embryonic life and progressed to tumoral progression linked to the ability of E-cadherin changing its phenotype from epithelial to mesenchymal features. Thus, E-cadherin is a pivotal element in the EMT process (promoting cellular invasion) and mesenchymal-epithelial one, respectively (promoting migration), ${ }^{24}$ and in vivo studies of E-cadherin have mainly been focused on EMT and MET in breast tumoral pathology. ${ }^{43,44}$ Experimental evidence indicates that low-level expression of E-cadherin is a marker of poor prognosis. ${ }^{25,26}$ Tang found that tumor size, highly undifferentiated tumors, positive LN status, loss of androgen receptor, and E-cadherin expression were significantly associated with DFS and the OS in TNBC. And patients with E-cadherin-reduced expression showed a shorter DFS and OS. ${ }^{26}$ Kashiwagi et al evaluated that low levels of E-cadherin were observed in 238 (41.5\%) of the 574 breast cancer cases and $90(73.2 \%)$ of the 123 TNBC cases. The reduction in E-cadherin was significantly frequent in cases of TNBC $(P<0.001)$ and LN metastasis $(P=0.032)$. The authors showed that TNBC patients with E-cadherinnegative expression predicted poorer outcomes in terms of OS and DFS than patients with E-cadherin-positive. ${ }^{45}$ In this study, we examined the expression of E-cadherin in 106 TNBC by immunohistochemistry. The study showed that reduced E-cadherin expression was detected in 68/106 (64.2\%) tumors. A reduction of E-cadherin expression was highly associated with LN metastasis $(P=0.027)$ and TNM stage $(P=0.022)$. This finding suggested that reduce of E-cadherin expression might be correlated to the development of LN metastases in breast cancer. Meanwhile, we found that E-cadherin-negative expression had a shorter OS and DFS. What is more, univariate and multivariate Cox regression showed that reduced expression of E-cadherin was an independent unfavorable prognostic factor. Thus, it is worthy to further evaluate its value as a follow-up parameter for the benefit of TNBC patients.

We further examined the correlation between NEDD9 expression and E-cadherin expression. The TNBC patients with NEDD9-positive/E-cadherin-negative expression had a significantly worse outcome than other groups. We found a negative correlation between NEDD9 and E-cadherin expressions, suggesting that NEDD9 may be involved in the invasion and metastasis of cancer cells through promotion of cell proliferation by the EMT process.

\section{Conclusion}

This study provides evidence that NEDD9 and E-cadherin expressions are associated with a poor prognosis of TNBC patients. Based on our results and other publications, NEDD9 and E-cadherin have an important role in TNBC progression, especially in its EMT process. Further studies are required to investigate the practical value of these new biomarkers and reveal its underlying mechanisms in the regulation of breast cancer progression.

\section{Disclosure}

The authors report no conflicts of interest in this work.

\section{References}

1. Boyle P. Triple-negative breast cancer: epidemiological considerations and recommendations. Ann Oncol. 2012;23(6):7-12.

2. Zhao S, Ma W, Zhang M, et al. High expression of CD147 and MMP-9 is correlated with poor prognosis of triple-negative breast cancer (TNBC) patients. Med Oncol. 2013;30(1):335.

3. Nielsen TO, Hsu FD, Jensen K, et al. Immunohistochemical and clinical characterization of the basal-like subtype of invasive breast carcinoma. Clin Cancer Res. 2004;10(16):5367-5374.

4. Dent R, Hanna WM, Trudeau M, Rawlinson E, Sun P, Narod SA. Pattern of metastatic spread in triple-negative breast cancer. Breast Cancer Res Treat. 2009;115(2):423-428.

5. Bauer KR, Brown M, Cress RD, Parise CA, Caggiano V. Descriptive analysis of estrogen receptor (ER)-negative, progesterone receptor (PR)-negative, and HER2-negative invasive breast cancer, the so-called triple-negative phenotype: a population-based study from the California cancer Registry. Cancer. 2007;109(9):1721-1728

6. Bidard FC, Conforti R, Boulet T, Michiels S, Delaloge S, André F. Does triple-negative phenotype accurately identify basal-like tumour? An immunohistochemical analysis based on 143 "triple-negative" breast cancers. Ann Oncol. 2007;18(7):1285-1286.

7. Cheang MC, Voduc D, Bajdik C, et al. Basal-like breast cancer defined by five biomarkers has superior prognostic value than triple-negative phenotype. Clin Cancer Res. 2008;14(5):1368-1376.

8. Prat A, Adamo B, Cheang MC, Anders CK, Carey LA, Perou CM. Molecular characterization of basal-like and non-basal-like triplenegative breast cancers. Oncologist. 2013;18(2):122-133.

9. O’Neill GM, Golemis EA. Proteolysis of the docking protein HEF1 and implications for focal adhesion dynamics. Mol Cell Biol. 2001; 21(15):5094-5108. 
10. O’Neill GM, Seo S, Serebriiskii IG, Lessin SR, Golemis EA. A new central scaffold for metastasis: parsing HEF1/Cas-L/NEDD9. Cancer Res. 2007;67(19):8975-8979.

11. Fashena SJ, Einarson MB, O’Neill GM, Patriotis C, Golemis EA. Dissection of HEF1-dependent functions in motility and transcriptional regulation. J Cell Sci. 2002;115(1):99-111.

12. Kim M, Gans JD, Nogueira C, et al. Comparative oncogenomics identifies NEDD9 as a melanoma metastasis gene. Cell. 2006;125(7): 1269-1281.

13. Sima N, Cheng X, Ye F, Ma D, Xie X, Lü W. The overexpression of scaffolding protein NEDD9 promotes migration and invasion in cervical cancer via tyrosine phosphorylated FAK and SRC. PLoS One. 2013;8(9):1-12.

14. Izumchenko E, Singh MK, Plotnikova OV, et al. NEDD9 promotes oncogenic signaling in mammary tumor development. Cancer Res. 2009; 69(18):7198-7206.

15. Kong C, Wang C, Wang L, et al. NEDD9 is a positive regulator of epithelial-mesenchymal transition and promotes invasion in aggressive breast cancer. PLoS One. 2011;6(7):e22666.

16. Tikhmyanova N, Golemis EA. NEDD9 and BCAR1 negatively regulate E-cadherin membrane localization, and promote E-cadherin degradation. PLoS One. 2011;6(7):1025-1048.

17. Zhang SS, Wu LH, Liu Q, Chen KS, Zhang XF. Elevated expression of NEDD9 is associated with metastatic activity in gastric cancer. Onco Targets Ther. 2015;8:633-640.

18. Chang JX, Gao F, Zhao GQ, Zhang GJ. Expression and clinical significance of NEDD9 in lung tissues. Med Oncol. 2012;29(4):2654-2660.

19. Budhu A, Forgues M, Ye QH, et al. Prediction of venous metastases, recurrence, and prognisis in hepatocellar cacinoma based on a unique immune response signature of the liver microenvironment. Cancer Cell. 2006;10(2):99-111.

20. Singh M, Cowell L, Seo S, O’Neill G, Golemis E. Molecular basis for HEF1/NEDD9/Cas-L action as a multifunctional co-ordinator of invasion, apoptosis and cell cycle. Cell Biochem Biophys. 2007;48(1): 54-72.

21. Law SF, O’Neill GM, Fashena SJ, Einarson MB, Golemis EA. The docking protein HEFI is an apoptotic mediator at focal adhesion sites. Mol Cell Biol. 2000;20(14):5184-5195.

22. Bui LC, Tomkiewicz C, Chevallier A, et al. Nedd9/Hef1/Cas-L mediates the effects of environmental pollutants on cell migration and plasticity. Oncogene. 2009;28(41):3642-3651.

23. Andrews JL, Kim AC, Hens JR. The role and function of cadherins in the mammary gland. Breast Cancer Res. 2012;14(1):203-213.

24. Chao YL, Shepard CR, Wells A. Breast carcinoma cells re-express E-cadherin during mesenchymal to epithelial reverting transition. Mol Cancer. 2010;9:179.

25. Tang D, Xu S, Zhang Q, Zhao W. The expression and clinical significance of the androgen receptor and E-cadherin in triple-negative breast cancer. Med Oncol. 2012;29(2):526-533.

26. Kashiwagi S, Yashiro M, Takashima T, et al. Advantages of adjuvant chemotherapy for patients with triple-negative breast cancer at Stage II: usefulness of prognostic markers E-cadherin and Ki67. Breast Cancer Res. 2011;13(6):R122.
27. Li P, Zhou H, Zhu X, et al. High expression of NEDD9 predicts adverse outcomes of colorectal cancer patients. Int J Clin Exp Pathol. 2014;7(5):2565-2570.

28. Prat A, Perou CM. Deconstructing the molecular portraits of breast cancer. Mol Oncol. 2011;5(1):5-23.

29. Perou CM. Molecular stratification of triple-negative breast cancers. Oncologist. 2011;16(1):61-70.

30. Adamo B, Anders CK. Stratifying triple-negative breast cancer: which definition(s) to use? Breast Cancer Res. 2011;13(2):105.

31. Harrell JC, Prat A, Parker JS, et al. Genomic analysis identifies unique signatures predictive of brain, lung, and liver relapse. Breast Cancer Res Treat. 2012;132(2):523-535.

32. Lin NU, Claus E, Sohl J, Razzak AR, Arnaout A, Winer EP. Sites of distant recurrence and clinical outcomes in patients with metastatic triple-negative breast cancer: high incidence of central nervous system metastases. Cancer. 2008;113(10):2638-2645.

33. Kalluri R, Weinberg RA. The basics of epithelial-mesenchymal transition. J Clin Invest. 2009;119(6):1420-1428.

34. Zeisberg M, Neilson EG. Biomarkers for epithelial-mesenchymal transitions. J Clin Invest. 2009;119(6):1429-1437.

35. Thiery JP. Epithelial-mesenchymal transitions in tumour progression. Nat Rev Cancer. 2002;2(6):442-454.

36. Thiery JP, Chopin D. Epithelial cell plasticity in development and tumor progression. Cancer Metastasis Rev. 1999;18(1):31-42.

37. Tiwari N, Gheldof A, Tatari M, Christofori G. EMT as the ultimate survival mechanism of cancer cells. Semin Cancer Biol. 2012;22(3): 194-207.

38. Mani SA, Guo W, Liao MJ, et al. The epithelial-mesenchymal transition generates cells with properties of stem cells. Cell. 2008;133(4) 704-715.

39. Mani SA, Yang J, Brooks M, et al. Mesenchyme Forkhead 1 (FOXC2) plays a key role in metastasis and is associated with aggressive basal-like breast cancers. Proc Natl Acad Sci U S A. 2007;104(24): 10069-10074.

40. Yu M, Smolen GA, Zhang J, et al. A developmentally regulated inducer of EMT, LBX1, contributes to breast cancer progression. Genes Dev. 2009;23(15):1737-1742.

41. Lu P, Wang ZP, Dang Z. Expression of NEDD9 in hepatocellular carcinoma and its clinical significance. Oncol Rep. 2015;33(5):2375-2383.

42. Kondo S, Iwata S, Yamada T, et al. Impact of the integrin signaling adaptor protein NEDD9 on prognosis and metastatic behavior of human lung cancer. Clin Cancer Res. 2012;18(22):6326-6338.

43. Hajra KM, Chen DY, Fearon ER. The SLUG zinc-finger protein represses E-cadherin in breast cancer. Cancer Res. 2002;62(6):1613-1618.

44. Cavallaro U, Schaffhauser B, Christofori G. Cadherins and the tumour progression: is it all in a switch? Cancer Lett. 2002;176(2):123-128.

45. Kashiwagi S, Yashiro M, Takashima T, et al. Significance of E-cadherin expression in triple-negative breast cancer. Br J Cancer. 2010;103(2): 249-255.
OncoTargets and Therapy

\section{Publish your work in this journal}

OncoTargets and Therapy is an international, peer-reviewed, open access journal focusing on the pathological basis of all cancers, potential targets for therapy and treatment protocols employed to improve the management of cancer patients. The journal also focuses on the impact of management programs and new therapeutic agents and protocols on

\section{Dovepress}

patient perspectives such as quality of life, adherence and satisfaction. The manuscript management system is completely online and includes a very quick and fair peer-review system, which is all easy to use. Visit http://www.dovepress.com/testimonials.php to read real quotes from published authors. 